\title{
INVESTIGACIONES
}

\section{Percepción docente de la naturaleza de la escucha pedagógica e implementación de las relaciones comunicativas en la educación secundaria}

\author{
Teacher perceptions of pedagogical listening and communicative relationships \\ in secondary education
}

\author{
Luisina Soria ${ }^{a}$, Antonio Giner Gomis ${ }^{b}$ \\ ${ }^{a}$ Universidad Nacional del Litoral, Argentina. \\ luisinasoria@hotmail.com.ar \\ ${ }^{b}$ Facultad de Educación. Universidad de Alicante, España. \\ a.giner@ua.es
}

\section{RESUMEN}

Esta investigación aborda el tipo de relaciones comunicativas que median entre docentes y alumnado en la etapa de Secundaria. Mediante un enfoque metodológico cualitativo, y a través de la tradición narrativa-biográfica han sido analizadas 20 narrativas distribuidas en 4 centros públicos en donde los docentes describen y significan las propias relaciones comunicativas con el alumnado destacando dimensiones y dificultades relevantes, su forma de gestionarlas y formación demandada.

A partir de los resultados, se visibiliza en este grupo de participantes la persistente proyección de un modelo comunicativo basado en una perspectiva comunicativa sustentada bajo un modelo de racionalidad instrumental, unidireccional que si bien transforma prácticas convencionales de enseñanza y aprendizaje puede correr el riesgo de reducir la relación comunicativa pedagógica a una interacción abstracta, desmotivadora pero especialmente pasivizadora del rol del alumnado al no alentar a una pedagogía de la participación como base de un aprendizaje igualitario, democrático y ético.

Palabras claves: Educación Secundaria, Enfoque Comunicativo, Relaciones Interpersonales, Desarrollo Profesional.

\section{ABSTRACT}

This study examines the type of communicative relations that exist between teachers and students in secondary education. Using a qualitative life story approach, we analysed 20 narratives obtained from teachers working in four state schools, in which they described and signified their communicative relations with students, highlighting relevant dimensions and difficulties, how they managed these and the training required.

The results revealed that participants persistently projected a communicative model based on a one-way model of instrumental rationality, which although it may transform conventional teaching and learning practices can also reduce teachers' communicative relations to an abstract interaction, rendering students passive and demotivated since this model does not foster participatory education based on egalitarian, democratic and ethical learning.

Key words: Secondary Education, Communicative Approach, Interpersonal Relationships, Professional Development. 
Estudios Pedagógicos XLVII N 1: 323-337, 2021

PERCEPCIÓN DOCENTE DE LA NATURALEZA DE LA ESCUCHA PEDAGÓGICA E IMPLEMENTACIÓN DE LAS RELACIONES COMUNICATIVAS EN LA EDUCACIÓN SECUNDARIA

\section{INTRODUCCIÓN}

\subsection{LAS RELACIONES COMUNICATIVAS EDUCATIVAS Y LA ESCUCHA DOCENTE COMO PROCESOS NATURALIZADOS Y SU NECESARIA REVISIÓN}

La persistencia de hábitos y rutinas en el ámbito profesional educativo que naturalizan e impregnan las prácticas cotidianas bajo el formato de comportamientos automatizados e inerciales, suelen limitar y ensombrecer experiencias de aprendizaje emancipadoras. La necesidad de problematizar y tomar consciencia de estas creencias latentes que colonizan nuestra acción profesional para poder crear nuevo sentido al quehacer profesional resulta insoslayable (Cabrera, 2003; Korthagen, 2016; Martin, Tarnanen y Tynjälä, 2018).

De otra parte, la transición del alumnado por la etapa de educación secundaria suele estar caracterizada por procesos críticos de desarrollo físico, psíquico, sexual, afectivo, etc. en su construcción identitaria (Monarca, Rappoport y Fernández, 2012; González, Vieira y Vidal, 2019), que demandan a sus docentes un eficiente acompañamiento en estos procesos, retándolos a un auténtico desafío pedagógico (Tenti Fanfani, 2010), tratando de posibilitar una epistemofilia permanente en el alumnado a través de la relación comunicativa inherente (Perrenoud, 2012; Recalcati, 2016).

Nuestra exploración se focaliza tanto en la comprensión de la naturaleza de la relación comunicativa educativa percibida por el docente de la E.S.O. analizando cómo la significan y el modo en que conceptualizan la escucha docente inserta en aquella (Segovia, Gallego y Rodríguez, 2013; Neira, Sierra y Pérez, 2018) con el fin de mejorar nuestra actuación profesional (Villalta, 2009; Funes, 2018; Tejedor, 2018).

Bajo estas premisas, hemos querido llevar a cabo nuestro análisis preguntando a los docentes sobre posibles situaciones de contacto pedagógico (Korthagen, Kim y Greene, 2013) para detectar qué tipo de escucha activan en el aula y sobre el significado que atribuyen a las relaciones de comunicación e implementación que despliegan en el aula. Para detectar posibles naturalezas tanto de la escucha pedagógica como de estas interacciones en el aula hemos adoptado tres perspectivas de aprendizaje: la cognitiva, (Pozo, 2011; Fleming, 2011), la sociocultural Wersch, 2005; Lawrence y Dodds, 2007) y la dialógica (Lave y Wenger, 1991; Akkerman y Meijer, 2011; Hennissen, Beckers y Moerkerke, 2017).

El contacto pedagógico al que nos referimos transforma una actuación comunicativa docente estrictamente académica (Ortega, 2017) en otra cuya calidad del encuentro va más allá de la interacción habitual y suele expresarse en términos de un estar docente empático y atento al alineamiento de sus propios valores con el del alumnado y a provocar en aquel mayor confianza en sí mismo y compromiso en su propio proceso de desarrollo personal. (Korthagen, Attema y Zwart, 2014; Ortega y Gárate, 2017).

\subsection{PERSPECTIVAS TEÓRICAS DEL APRENDIZAJE E IMPLICACIONES SOBRE LA COMPETENCIA COMUNICATIVA INCARDINADA EN ELLOS}

\subsubsection{Perspectiva Cognitiva y modelo pedagógico constructivista}

La perspectiva cognitiva, subsume particularmente los diversos trabajos de la Escuela Piagetana o de Ginebra, (Piaget, Bruner, Kholberg, Flavell) y promueve un 
constructivismo cognitivo a fin de desarrollar un pensamiento representacional en el estudiante con mediación del docente y de los materiales pertinentes (Pons y Serrano, 2011; Villalta, Martinic, Assael y Aldunate, 2018). Sin menoscabar el indudable avance, logros y cambios que han supuesto los modelos pedagógicos constructivistas respecto de la pedagogía tradicional (Offir, 2012), la interacción comunicativa puede quedar circunscrita a situaciones tríadicas: docente, alumnado y materiales (Fernández, Tusset, Pérez y García, 2013). Y la comunicación, frecuentemente canalizada en un único sentido: promover un aprendizaje de contenidos asépticos, descontextualizados y eviscerados del mundo emocional, corporal o ético. En consonancia con Pozo (2017) las actividades curriculares suelen integrarse bajo la categorización AAA (abstracta, arbitraria y amodal), desplazando a las categorizadas como EEEE (encarnadas, enactivas, encajadas o situadas y extendidas) y pueden dificultar al alumnado a constituirse como un actor activo y crítico dentro de un contexto escolar inevitablemente vertebrado por relaciones de poder (Cros, 2002; Helgevold, 2016).

\subsubsection{Perspectiva Sociocultural y modelo pedagógico socio-constructivista}

Emergiendo desde la psicología rusa (Vigotsky, 1978; Leontiev, Luria y Vigotsky, 2004, Guitar, 2011), cobra importancia la comprensión de los procesos de desarrollo cognitivo a partir del aprendizaje mediado cultural e históricamente en contra de todo reduccionismo biológico y conductismo metodológico; cuestionándose así el cognitivismo más radical piagetano al no tener en cuenta aquellas dimensiones y dinámicas interactivas socioculturales que impregnan los diversos contextos de desarrollo humano (Chaves, 2001; Bronfenbrenner, 2005). Emerge significativo el aprendizaje situado y funcional enmarcado culturalmente (Del Rio y Álvarez, 2002; Damianova y Sullivan, 2011), aunque, al menos en el contexto escolar no consigue desplazar en términos pedagógicos al modelo hegemónico psicológico cognitivista más de corte empirista y que parte del supuesto de una correspondencia entre conocimiento y realidad, observable desde fuera y asimilable mediante una comprensión objetiva inequívoca y adherido con fuerza al sistema educativo. Bajo este modelo, la interacción pedagógica en el aula puede seguir inserta en dinámicas de comunicación opacas a visibilizar relaciones de poder y exclusión evasivas a problematizar una construcción más simétrica o participada del saber. La participación y consideración de los diversos agentes educativos, locales, situados y contextualizados, con agencia para tomar decisiones compartidas o negociadas en la educación puede seguir excluyendo al alumnado de una auténtica co-construcción de su propio aprendizaje.

\subsubsection{Perspectiva dialógica y el modelo Comunidades de aprendizaje}

Enraizada en un enmarcamiento diverso como la psicología cultural (Pizzinato, 2009), la narratividad (Cladinin, 2007; Rivas-Flores, 2014), la teoría del lenguaje de Bakhtín, 1998, 2015) o la teoría de la acción comunicativa de Habermas (1989, 2004), alienta procesos relacionales democráticos y descolonizantes en la constitución identitaria. De tal suerte que las Comunidades de Aprendizaje como modelo dialógico del aprendizaje coherente con aquella, enfatiza un diálogo igualitario y argumentativo como herramienta necesaria para construir consensos (Prados, Gómez del Castillo y Aguilera, 2016), y desafiante de una pedagogía que instrumentaliza el formato interactivo habitual para 
conducir al alumnado a los estándares curriculares (Matusov, 2015). Las Comunidades de Aprendizaje, (Aubert, Flecha, García y Racionero, 2010) como proyección teóricopráctica de esta perspectiva y a través de sus actuaciones de éxito (Fernández, Garvin y González, 2012) tratan de empoderar a las diferentes agencias decisoras, familias, alumnado o voluntariado, apostando por una justicia curricular que supere desigualdades sociales, aportando cohesión social y gestión distribuida de poder en los centros. (Aguilar, Padrós, Pulido y Alonso, 2010; Chocarro de Luis y Sáenz de Jubera, 2016).

El concepto aprendizaje dialógico es posibilitado mediante estas comunidades de aprendizaje al desarrollarse un concepto de escucha particularmente ético, respetuoso y más democrático (Freire, 2004; Fernández, Garvín y Gonzalez, 2012; Sousa, 2017; Ruhalahti y Aarnio, 2018; Zhao y Biesta, 2012), abierto a la otredad (Gehrke, 2009), a la incertidumbre (Arndt, 2017) o a limitar enjuiciamientos reactivos inerciales en la comunicación cotidiana (Beard, 2009).

Desde este marco teórico, hemos abordado las siguientes cuestiones de investigación:

1. ¿Qué competencias profesionales y relevantes perciben los docentes como necesarias para abordar una labor óptima en la Educación Secundaria Obligatoria (E.S.O)?

2. ¿Qué significado le otorgan estos mismos participantes a la escucha docente o escucha activa en su trabajo diario como procedimiento ineludible para captar necesidades y demandas globales del alumnado en el aula?

3. ¿Cómo describen los docentes los momentos personales de contacto pedagógico,

4. ¿Qué dimensiones internas destacan en las relaciones comunicativas pedagógicas con sus estudiantes?

5. ¿A qué dificultades se enfrenta el colectivo docente en las relaciones comunicativas con su alumnado? ¿Cuáles son los modos de resolver y gestionar esas dificultades?

6. ¿Qué aprendizajes en opinión de los docentes son necesarios para desarrollar una escucha activa y ética con su alumnado?

\section{MÉTODO}

El estudio llevado a cabo es de carácter cualitativo- descriptivo, (Rodriguez-Hoyos, CalvoSalvador y Haya-Simón, 2014), inserto en la tradición narrativa biográfica, capaz de captar en estos relatos percepciones docentes susceptibles de ser analizadas y comprendidas (Giner, Iglesias y Lozano, 2018; Oliveira, Santos Mafra, Amorim de Oliveira y Sandes, 2018) y pretende más una comprensión que una generalización de sus resultados poniendo énfasis en lo individual e inductivo (Sánchez, 2018).

\subsection{PARTICIPANTES}

La muestra de esta investigación queda constituida por un total de 20 participantes, seleccionados por un muestreo no probabilístico e intencional (Otzen y Manterola, 2017) y distribuidos en cuatro centros de Secundaria; los docentes desempeñan su trabajo en Centros públicos de la provincia de Alicante. Un 35\% se autoperciben como mujeres y un $65 \%$ como varones. En cuanto a las edades del profesorado, un $15 \%$ queda situado entre 
25 y 35 años; un $45 \%$ entre 36 y 46 años; un 30\% entre 47 y 57 años y un $10 \%$ en personas con edades entre 58 a 68 años; un $38,70 \%$ de las participantes imparte en $1^{\circ} \mathrm{ESO}$, un $12 \%$ en $2^{\circ}$ ESO, un $12,90 \%$ en $3^{\circ}$ de la ESO y un $32,25 \%$ en $4^{\circ}$ de la ESO. En cuanto a su experiencia profesional, un $15 \%$ se sitúa entre 21 y 30 años, un $60 \%$ en el rango de 11 a 20 años y un $25 \%$ en el de 0 a 10 a años.

\subsection{INSTRUMENTO DE RECOGIDA DE DATOS}

La entrevista semiestructurada (Kvale, 2011; Romero, Figuera Freixa y Llanes, 2019) nos ha permitido recopilar, coherentemente con el enfoque narrativo, la información necesaria para analizar el sentido que le otorgan los docentes a la comunicación y escucha en el aula.

\subsection{PROCEDIMIENTO}

Las entrevistas se realizaron de forma presencial en los centros, garantizando el anonimato y la confidencialidad de los participantes. También se dio la opción de contestarla, a quienes así optaron, mediante un formulario preparado con la aplicación de Google Forms. En ambas modalidades se incluyeron las mismas preguntas.

\subsection{GESTIÓN DE LOS DATOS NARRATIVOS.}

Las entrevistas realizadas se registraron mediante un código alfanumérico y para reducir el volumen narrativo utilizamos la aplicación informática AQUAD Six 6, Analisys of Qualitative Data, bajo soporte Windows (Huber y Gurtler, 2012) que nos ha facilitado simultáneamente la sistematización y codificación de los datos (Angrosino, 2012.). Tras reiteradas lecturas para ganar fiabilidad inter-observador, y mediante un proceso mixto inductivo-deductivo establecimos consensuadamente los criterios de codificación para construir el correspondiente mapa de códigos validado mediante triangulación por tres expertos en el área D.O.E. Las seis cuestiones de investigación han conformado el eje vertebrador y analítico de las narrativas docentes. Así, la primera cuestión de investigación proyecta la temática Competencias Relevantes Generales. La segunda de las cuestiones identifica la temática Significado de escucha docente; la tercera cuestión explora la Percepción Docente del Contacto Pedagógico; La cuarta de las temáticas aborda las Dimensiones Internas Relevantes en la Comunicación de Aula; la quinta cuestión explora las Dificultades en sus Relaciones Comunicativas con el Alumnado y finalmente, la sexta cuestión indaga sobre la Formación Específica Demandada para gestionar mejor el campo comunicacional en el aula. Tras reiteradas lecturas de las narrativas docentes, elaboramos el correspondiente mapa de códigos, adscritos a cada una de las temáticas y definidos tanto por su contenido como por su frecuencia en el conjunto de cada temática analizadas. A modo de ejemplificación de la primera temática, Competencias Relevantes Generales, las categorías o códigos emergentes fueron: 1.1 competencia TransversalCurricular (importancia de una buena gestión en el aula para dirigir al alumnado hacia el aprendizaje curricular); 1.2 Competencia metodológica (capacidad de implementar didácticamente en el aula el conocimiento disciplinar), 1.3 Competencia ética (la relevancia en la enseñanza de valores particulares como la puntualidad, responsabilidad, compromiso, etc.). 
Estudios Pedagógicos XLVII Nº 1: 323-337, 2021

PERCEPCIÓN DOCENTE DE LA NATURALEZA DE LA ESCUCHA PEDAGÓGICA E IMPLEMENTACIÓN DE LAS RELACIONES COMUNICATIVAS EN LA EDUCACIÓN SECUNDARIA

\section{RESULTADOS}

\subsection{COMPETENCIAS PROFESIONALES Y EDUCATIVAS RELEVANTES QUE IDENTIFICA EL PROFESORADO PARTICIPANTE}

Destaca por su alta presencialidad en las narrativas de los participantes la competencia codificada como Transversal-Curricular significando la necesidad de gestionar y dirigir al alumnado hacia los aprendizajes curriculares en cualquier materia académica: "Capacidad de gestionar grupos de alumnos, habilidades y capacidad para que los alumnos aprendan". (P012). Una segunda competencia, la metodológica, menor en su frecuencia, refiere el modo de implementar didácticamente tanto contenidos disciplinares como la gestión conflictual en la clase: "Competencia didáctica, gestión de convivencia, TIC, resolución de conflictos, etc." (P013). Finalmente, y de forma casi simbólica, ha emergido la competencia ética con la que refieren la importancia de la enseñanza de valores como el compromiso, puntualidad, la responsabilidad, etc.: "La forma en la que se valora el aprendizaje humano. Puntual, responsable, asume compromiso." (P002).

\subsection{EL SIGNIFICADO DE LA ESCUCHA DOCENTE SEGÚN LA PERCEPCIÓN DE LOS PARTICIPANTES}

Prevalece el código escucha curricular con la que el docente, actor principal, sujeto con poder y saber, posibilita adecuar los contenidos curriculares al alumnado: "El docente ha de estar atentos a todos los mensajes que le llegan desde el alumnado para redirigir la actividad docente." (P008). En algún caso, perciben la escucha con cierta apertura dialógica hacia el alumnado, pero, sigue orientada enfáticamente hacia la enseñanza:

Ser capaz de dialogar con ellos es imprescindible, pero para que exista diálogo ha de haber escucha por parte de ambas partes y lógicamente se ha de comenzar por el/la profesorla que ha de mostrar el camino y ser capaces de "enseñar". (P003)

El código emergente más débil presencialmente, refiere la escucha para detectar dimensiones actitudinales personales de alumnado consideradas relevantes para, de nuevo garantizar el éxito académico: "Actualmente una gran parte del alumnado en esta etapa tiene importantes problemas de concentración, atención y disponibilidad para estudiar." (P009).

\subsection{LA PERCEPCIÓN DEL CONTACTO PEDAGÓGICO}

Mediante este concepto hemos propuesto a los participantes una descripción de estos momentos "cumbre" en donde comunicación educativa ha podido establecerse de una forma particularmente intensa y recíproca.

A través del código contacto contingente, el de mayor presencialidad, recuerdan situaciones educativas en donde el interés y compromiso que los estudiantes manifestaron emergió por motivaciones aleatorias: "Son momentos en los que notas que conectas, que de pronto te están "dejando entrar". No sé muy bien cuál es la clave. A veces es porque el tema les interesa (es difícil saber cual les va a interesar." (P001). Con una acusada menor intensidad, y mediante el código contacto motivacional, enfatizan la clave de una óptima 
activación actitudinal en el alumnado en relación a los objetivos curriculares: "El docente ha sido capaz de despertar el interés del alumnado (en la materia)." (P004). En tercer lugar, mediante el código contacto práctico manifiestan como posible causa el desarrollar actividades de aula no habituales: "Cuando se trabaja por proyectos y se plantean ejercicios que requieran ser divergentes. Trabajos cooperativos, debates, salidas extraescolares, tertulias dialógicas." (P013). Final y residualmente con el código contacto empático atribuyen la causa del contacto pedagógico a un clima de aula distendido que posibilita una particular e interesante interacción docente- discente: "Se ha establecido previamente en clase un clima de tranquilidad y empatía mutua. Hay un clima de colaboración en clase entre los alumnos y el profesor." (P008).

\subsection{DIMENSIONES INTERNAS QUE DESTACAN EN LAS RELACIONES COMUNICATIVAS PEDAGÓGICAS}

A través del código dimensión ética, destacan prevalentemente la importancia de transmitir valores claves facilitadores de óptimas relaciones comunicativas: "Actitud de respeto hacia el/la profesora. Hacia los compañeros y hacia el material (aula, libros...)." (P010). Con menor intensidad y con el código dimensión lingüística describen la importancia de la expresión y comunicación lingüística: "Saber todas las lecturas comprensivas... expresión oral. Saber argumentar y expresar todos los aprendizajes. Saber comunicarse." (P005). El código menos visibilizado refiere la dimensión comportamental otorgando relevancia a los hábitos de trabajo, orden, etc. como facilitadores comunicacionales: "Orden, respeto trabajo en equipo, límites, podemos bromear pero sin romper los límites de trabajo" (P019).

\subsection{DIFICULTADES EN LAS RELACIONES COMUNICATIVAS Y MODOS DE GESTIONARLAS}

Con una alta presencia del código dificultades individuales del alumnado, y de manera explícita, señalan en el alumnado debilidades comunicativas: "Falta de interés y motivación; resistencia a cambiar hábitos y les cuesta y evitan." (P001). Distanciado considerablemente del primer código y rotulado como Contenidos contradictorios, refieren la existencia del vacío que separa objetivos y contenidos curriculares con la vida cotidiana del alumnado actual: Alejamiento entre lo que se enseña y el día a día del alumno." (P004). A la par, con el código dificultades estructurales del sistema educativo, refieren las excesivas demandas de un sistema educativo que sobrepasa con sus exigencias al docente: "La falta de tiempo, el cansancio y la sensación de no poder abarcar aquello que se me plantea delante. Creo que la cantidad de tareas y número de personal al que atendemos nos sobrepasa." (P003). Como propuestas de gestión de estas mismas dificultades, hacen prevalecer aquellas que resuelvan la gestión conflictual y emocional en el alumnado: "[...la necesidad de implementar]... programas y estrategias de habilidades sociales, inteligencia emocional y resolución de conflicto." (P013). En un segundo lugar señalan la modificación del rol docente habitual con el fin de conseguir una actitud más empática con el alumnado: "Respetando primero al alumnado... que sepa que nos ponemos en su lugar. Y argumentarle el hecho de cambiar su actitud y comportamiento." (P005). Por último, explicitan mínimamente una gestión a través del diálogo como modo de resolver las dificultades comunicativas: "Creo que el diálogo es fundamental y aunque ellos no lo vean ahora, tal vez en un futuro sí que recapaciten sobre todo lo que decimos aquî” (P007). 
Estudios Pedagógicos XLVII N 1: 323-337, 2021

PERCEPCIÓN DOCENTE DE LA NATURALEZA DE LA ESCUCHA PEDAGÓGICA E IMPLEMENTACIÓN DE LAS RELACIONES COMUNICATIVAS EN LA EDUCACIÓN SECUNDARIA

\subsection{FORMACIÓN EN RELACIÓN AL ASPECTO COMUNICATIVO Y DIALÓGICO}

Las participantes manifiestan preferentemente dos campos de intereses; el más señalado lo hemos definido mediante el código inteligencia emocional: "Sí. Inteligencia emocional para trabajar la resolución de conflictos, autoestima, autoconcepto." (P014). En segundo lugar, distanciado del primero, demandan la actualización de ciertas competencias favorecedoras de la comunicación: "Habilidades comunicativas: escucha activa, empatía, negociación, conflictos, respeto, persuasión, comunicación no verbal, individual y colectiva” (P012).

\section{DISCUSIÓN Y CONCLUSIONES}

\subsection{COMPETENCIAS PROFESIONALES Y RELEVANTES QUE PERCIBEN LOS DOCENTES COMO NECESARIOS PARA ABORDAR UNA LABOR ÓPTIMA EN LA EDUCACIÓN SECUNDARIA OBLIGATORIA}

Nuestro trabajo sugiere que las competencias declaradas como más relevantes quedan vinculadas con una perspectiva metodológica comunicativa fuertemente cognitivista, técnica y "aséptica" del acto educativo (Pozo, 2017). Circunscritas a situaciones triádicas, donde alumnado, docentes y actividades dejan intocables las relaciones convencionales de poder (Fernández, Tuset, Pérez y García, 2013). Paradójicamente la competencia ética, referida al desarrollo de un compromiso social y a la promoción de comportamientos cooperativos que faciliten una interacción social y solidaria entre las personas es visibilizada de manera simbólica y residual (García, 2010). Observamos aquí un intenso modelaje del alumnado orientándolo particularmente hacia un sujeto escolar más receptivo que participante (Helgevold, 2016; Lechuga y Ramírez, 2015; Carvajal, 2019) y a una educación más empobrecedora que creadora, pacífica o cooperativa (Argudín, 2001; Castillo y Cabrerizo, 2010) invisibilizando una dimensión ética y política en el sentido aristoteliano y difuminando así una escuela de rostro más humano (Ortega y Gárate, 2017).

\subsection{EL SIGNIFICADO QUE LE OTORGAN LOS DOCENTES A LA ESCUCHA DOCENTE O ESCUCHA ACTIVA EN SU TRABAJO DIARIO}

La acentuación de una escucha que tramita permanentemente un proceso técnico curricular antes que una gestión de las inevitables situaciones dilemáticas en la enseñanza evade las complejas situaciones relacionales de acto comunicativo pedagógico. Llama la atención la inexistencia de reflexiones que pongan el foco de atención en la escucha introspectiva docente para revisar su propia práctica (Gehrke, 2009) pues el interés de su escucha se dirige a reajustar la práctica curricular identificando los errores del alumnado, minimizando aquella otra naturaleza posibilitadora de una co-construcción del propio proceso de aprendizaje en el que se hallan inmersos ambos (Ortega, 2017); bajo el manto protector de una racionalidad comunicativa-disciplinar, la escucha docente practicada proyecta, según sostiene Cros, (2002), la sombra de un acto comunicativo básicamente unidireccional.

La ausencia en estos relatos de una escucha empática docente capaz de crear sentido y significados nuevos en el escenario formativo, educativo y ético es manifiesta (Freire, 2004; Fernández et al., 2012; Sousa, 2017; Ruhalahti y Aarnio, 2018). 


\subsection{PERCEPCIÓN DOCENTE SOBRE EL CONTACTO PEDAGÓGICO}

La dificultad para describir y explicar argumentativamente en sus narrativas docentes qué condiciones pueden hacer emerger un contacto pedagógico auténtico es manifiesta (Korthagen, Attema y Zwart, 2014) y seguimos percibiendo en sus relatos una perspectiva comunicativa fuertemente cognitiva y unidireccional pues el diseño de un ambiente estimulante y motivador en el que el estudiante pueda desarrollar los objetivos educativos es vista mayoritariamente como una responsabilidad exclusiva docente (Fernández, Tuset, Pérez y García, 2013). La inexistencia en estas narrativas de reflexiones que señalen otros aspectos facilitadores del contacto pedagógico como la apertura a lo desconocido, (Arndt, 2017), una actitud ética para recibir a la otredad (Gehrke, 2009; Lipari, 2009), el silencio o suspensión de juicio egótico (Beard, 2009) o unas relaciones más democráticas (Penman y Turnbull, 2012), podrían conformar puntos ciegos en sus narrativas limitando una mejor comprensión epistemológica sobre el propio quehacer docente (Leijen, Allas, Pedaste y Knezic, 2015; Phillips y Napan, 2016) y contribuyendo al desarrollo de un docente más consciente de sus creencias (Korthagen, 2004; Korthagen, 2010; Nuñez y Fontana, 2009; Aubert et al., 2010).

\section{4. ¿QUÉ DIMENSIONES INTERNAS DESTACAN EN LAS RELACIONES COMUNICATIVAS PEDAGÓGICAS CON SUS ESTUDIANTES?}

Priorizando la enseñanza de ciertos valores éticos como el del respeto mutuo, tanto hacia el docente como entre el alumnado o hacia el medio material, se resuelve más como una experiencia vivencial unidireccional impuesta que como fruto de una pedagogía de la co-participación, co-constructora del conocimiento académico puesto que desarrollar y ejercitar cooperativamente una ciudadanía democrática y respetuosa implica procesos de aprendizaje en donde se ofrezcan condiciones que garanticen dinámicas participativas de diálogo igualitario y creación de nuevo sentido con los que posibilitar una trasformación de las relaciones de poder que no se han visibilizado, hasta el momento, en sus narrativas. para la resolución de problemas (Lozano, Ballesta, Castillo y Cerezo, 2018).

Congruentemente, al hacer prevalecer la internalización de hábitos conductuales y el desarrollo de la dimensión lingüística y expresiva en los estudiantes, junto a "la enseñanza de valores" sugerimos que las narrativas interpretadas siguen formateando los aspectos comunicacionales educativos bajo criterios técnicos y racionales- académicos como señalan Sarceda y Rodicio (2018). Adicionalmente observamos que el sujeto sobre el que recae la responsabilidad principal comunicativa está focalizado persistentemente en el estudiante y el docente sigue evadiéndose como actor susceptible de ser problematizado promoviendo una perspectiva que entiende la construcción del saber académico casi de exclusiva responsabilidad individual del alumnado y de su autorregulación como señala el trabajo de Villalta, Martinic, Assael y Aldunate, (2018).

Tampoco visibilizan reflexiones sobre la empatía, el silencio o el compromiso (Castellá, Comelles, Cros y Santasusana, 2006) como indicadores de la capacidad comunicativa del docente para mantener un adecuado balance entre distancia y proximidad discursivas. En este sentido, los trabajos de Aubert et al. (2010), Fernández et al. (2012), Aguilar, Padrós, Pulido y Alonso, (2010), y Ruhalahti y Aarnio (2018) siguen mostrando que el diálogo igualitario en la interacción cara a cara es un reto pendiente para la institución educativa. 


\subsection{LAS DIFICULTADES QUE ENFRENTA EL COLECTIVO DOCENTE EN LAS RELACIONES COMUNICATIVAS CON SU ALUMNADO Y LOS MODOS DE GESTIONAR DICHAS DIFICULTADES}

Asuntos como la falta de hábitos de atención en el alumnado, la brecha entre los contenidos funcionales prácticos y los teóricos o la percepción de un sistema que demanda un volumen de tareas docentes que los agota y extenúa con la sensación de no poder abarcar lo que se les solicita institucionalmente proyectan dificultades invariablemente externalizadas (Argudín, 2001) y desde un posicionamiento dialógico esto puede conducirnos a gestiones simplistas y reduccionistas de las dinámicas comunicativas (Morin, 2009) si el docente no está dispuesto a cuestionar sus creencias (Choi Fung Tam, 2015). Efectivamente, en ningún momento se autoperciben como factor a problematizar revisando sus propias pautas de pensamiento y relaciones comunicacionales.

La resolútica docente se centra así en el método como dimensión técnica y principal capaz de modificar situaciones y mantiene al docente como el elemento nuclear, mediador y directivo para el desarrollo del aprendizaje del estudiante dentro del entramado relacional escolar y tradicional (Agra et al., 2019). El docente sigue ocupando el lugar de privilegio y de trasmisión, relegando el diálogo a la última de las categorías o procedimientos con la que gestiona las dificultades comunicativas. obviando así una perspectiva dialógica o de Comunidades de Aprendizaje promotora de una actitud de escucha (León, 2019) que comprometa a estudiantes como co-partícipes de sus aprendizajes (Dziubinski, 2014; Álvarez, 2017; Coiduras, Balsells, Alsinet, Urreal, Guadix y Belmonte, 2014).

\subsection{LOS APRENDIZAJES QUE LOS DOCENTES CONSIDERAN NECESARIOS PARA DESARROLLAR UNA ESCUCHA ACTIVA Y ÉTICA PARA CON SU ESTUDIANTADO}

En consonancia con los resultados previos, y debido a esta lógica racional cognitiva y comunicativa, persistente y latente, sus intereses formativos señalan en un primer lugar metodologías, competencias y contenidos específicos con los que comprender y gestionar técnicamente el mundo emocional del alumno, continuando/ubicando al docente en un rol activo y al estudiante en un rol pasivo, Fernández et al. (2013). En un segundo lugar destacan aquellos contenidos y competencias que contribuyen a la comunicación proponiendo contenidos fuertemente psicologizados como la escucha activa, comunicación no verbal, gestión de conflictos, etc. y que concuerdan con los trabajos de Menéndez (2018) y Prieto (2018) quienes destacan la preocupante primacía reduccionista de una psicologización en la educación que constriñe las relaciones comunicativas a herramientas lingüísticas y emocionales para gestionar los procesos de aprendizajes.

Concluimos con la necesidad de revisar y tomar consciencia de la naturaleza y calidad de las relaciones comunicativas y escucha en esta etapa (Pagès, 2016), para evitar la colonización de un modelo pedagógico basado en una acusada racionalidad instrumental comunicativa desatendiendo otros parámetros que hacen posible diálogos y conversaciones intersubjetivas educativas más auténticas y completas. Específicamente, pensamos que conviene desarrollar una saludable perspectiva crítica a un modelo estrictamente cognitivista encapsulado en relaciones comunicativas desafectas de un mundo más contextualizado y emocional. Coherentemente con esta perspectiva, los procesos de escucha se instrumentalizan como procesos técnicos y poco empáticos que distancian motivacionalmente al alumnado. Cobra sentido así que la percepción docente sobre aquellas 
situaciones especialmente ricas e inspiradoras para ambos, generalmente ocasionales, como las del contacto pedagógico, no se describen haciendo uso de consideraciones sobre actitudes docentes de apertura a lo imprevisto, a la otredad, al valor del silencio en la comunicación o las relaciones latentes de poder siempre presentes en las interacciones entre ambos actores. La priorización y reducción de valores éticos en la enseñanza a conductas de respeto fundamentalmente acompasadas con el interés por una comprensión lingüística o de hábitos de trabajo como dimensiones internas a las relaciones comunicativas reiteran un pensamiento modelador de un formato comunicativo de hondo calado tecnocrático y cegado a ver otras consideraciones vitales en el alumnado. Consecuentemente tanto las dificultades como la formación demandada, evade focalizar sus pretensiones en una revisión interna de las propias creencias docentes. Indudablemente, y finalizamos con ello, necesitamos problematizar nuestras concepciones más arraigadas de comunicación porque precisamente desarrollamos nuestra labor docente mediante esa asombrosa capacidad que nos marca la diferencia como especie.

\section{REFERENCIAS BIBLIOGRÁFICAS}

Aguilar, C., Padrós, M., Pulido, M. A. y Alonso, M. J. (2010). Lectura dialógica y transformación de las comunidades de aprendizaje. Revista interuniversitaria de formación del profesorado, (67), 31-44.

Agra, G., Formiga, N. S., Oliveira, P. S. D., Costa, M. M. L., Fernandes, M. D. G. M. \& Nóbrega, M. M. L. D. (2019). Analysis of the concept of Meaningful Learning in light of the Ausubel's Theory. Revista brasileira de Enfermagem, 72(1), 248-255.

Akkerman, S. F. y Meijer, P. C. (2011). A dialogical approach to conceptualizing teacher identity. Teaching and Teacher Education, 27, 308-319.

Álvarez, C. (2017). La mejora de la participación de la comunidad en la escuela con grupos interactivos. Revista Complutense de Educación, 28(3), 815-828.

Angrosino, M. (2012). Etnografía y observación participante en investigación cualitativa. Madrid: Morata

Argudín, Y. (2001). Educación basada en competencias. Magistralis, 20, 39-61. Recuperado el 8 de noviembre de 2018 desde https://bit.ly/2I1k9Sb

Arndt, S (2017). Dialogic ruptures: an ethical imperative. Educational Philosophy and Theory, 49, 909-921. DOI: https://doi.org/10.1080/00131857.2015.1135776

Aubert, A., Flecha, A. \& García, C. F. R. y Racionero, S. (2010). Aprendizaje Dialógico en la Sociedad de la Información.

Bakhtín, M. M. (2015). Yo también soy. (Fragmentos sobre lo otro). Buenos Aires: Ediciones Godot. . (1998). Estética de la creación verbal. España: Siglo veintiuno editores.

Beard, D. (2009). A broader understanding of the ethicss of listening:philosophy, cultural studies, media studies and the ethical listening subject. International Journal of listening 23(1), 7-20. DOI: http://doi.org/10.1080/10904010802591771.

Bronfenbrenner, U. (2005). Making human beings human. London: Sage Publications

Cabrera, J. D. (2003). Discurso docente en el aula. Estudios pedagógicos, (29), 7-26. DOI: 10.4067/ S0718-07052003000100001

Carrapato, J., Castanheira, E. y Placideli, N. (2018). Percepções dos profissionais de saúde da atenção primária sobre qualidade no processo de trabalho. Saúde e Sociedade, 27(2), 518-530. https:// dx.doi.org/10.1590/s0104-12902018170012

Castellá, J.M., Comelles, S., Cros, A. y Santasusana, M.V. I. (2006). "Yo te respeto, tú me respetas". Estrategias discursivas e imagen social en la relación comunicativa en el aula. Infancia y Aprendizaje, 29(1), 31-49. DOI: 10.1174/021037006775380902 
Estudios Pedagógicos XLVII N 1: 323-337, 2021

PERCEPCIÓN DOCENTE DE LA NATURALEZA DE LA ESCUCHA PEDAGÓGICA E IMPLEMENTACIÓN DE LAS RELACIONES COMUNICATIVAS EN LA EDUCACIÓN SECUNDARIA

Castillo, S. y Cabrerizo, J. (2010). Evaluación educativa de aprendizajes y competencias. Madrid: Pearson educación S.A.

Chaves, A. (2001). Implicaciones educativas de la teoría sociocultural de Vigotsky. Educación, 25(2), 59-65.

Chocarro de Luis, E. y Sáenz de Jubera, M. (2016). Grupos interactivos: estrategia para la mejora de la convivencia, la participación y el aprendizaje. Revista Complutense de Educación, 27(2) 585-601.

Choi Fung Tam, A. (2015). The role of a professional learning community in teacher change: a perspective from beliefs and practices. Teachers and Teaching, 21(1), 22-43. DOI: $10.1080 / 13540602.2014 .928122$

Clandinin, D. J. (2007). (Edit.). Handbook of narrative inquiry. Mapping a methodology. California: Sage Publications, Inc.

Coiduras, J. L., Balsells, M. A., Alsinet, C., Urreal, A., Guadix, I. y Belmonte, O. (2014). La participación del alumnado en la vida del centro: una aproximación desde la comunidad educativa. Revista Complutense de Educación, 27(2), 437-456.

Cros, A. (2002). Elementos para el análisis del discurso en las clases. Cultura y Educación, 14(1), 81-97. DOI: 10.1174/113564002317348138

Damianova, M. K., y Sullivan, G. B. (2011). Rereading Vygotsky's Theses on Types of Internalization and Verbal Mediation. Review of General Psychology, 15(4), 344-350. https://doi.org/10.1037/ a0025627

Del Río, P. y Álvarez, A. (2002). From activity to diectivity: the question of involvement in education. In G. Wells y G. Claxton (Ed.). Learning for life in the 21St: sociocultural Perspectives on the future of Education (pp. 59-72), Oxford: Blackwell Publishers

Duschatzky, S. (2017). Política de la escucha en la escuela. Buenos Aires: Paidós.

Dziubinski, J. P. (2014). Does feeling part of a learning community help students to do well in their A-levels? Exploring teacher-student relationships. Research in PostCompulsory Education, 19(4), 468-480. DOI: 10.1080/13596748.2014.955641

Fernández, M. T, Tuset, A. M, Pérez, R. E. y García, C. (2013). Prácticas educativas y creencias de profesores de secundaria pertenecientes a escuelas de diferentes contextos socioeconómicos. Perfiles Educativos, 35(139), 40-59.

Fernández, S., Garvin, R. y González, V. (2012). Tertulias pedagógicas dialógicas: con el libro en la mano. REIFOP, 15(4), 113-118.

Fleming, T. (2011). Models of lifelong learning: an overview. En A. M. london (Ed.). The Oxford handbook of lifelong learning. New York: Oxford University Press.

Freire, P. (2004). La educación como práctica de la libertad. Argentina: Siglo XXI.

Funes, M. J. (2018). Erving Goffman, su perfil y su obra. Tendencias Sociales. Revista de Sociología, (2), 5-22.

García, E. (2010). Competencias éticas del profesor y calidad de la educación. REIFOP, 13(4), 21-41.

Gehrke, P. J. (2009). Introduction to listening, ethics, and dialogue: between the ear and the eye: A sinaesthetic introduction to listening ethics. International journal of listening, 23(1). doi: https:// doi.org/10.1080/10904010802631023

Giner, A., Iglesias, M. y Lozano, I. (2018). Classical Myth in the University: A Contribution to Professional Teacher Development. International Education Studies, 11(12), 1-11.

González, D.; Vieira, M.J. y Vidal, J. (2019). Variables que influyen en la transición de la educación Primaria a la educación Secundaria obligatoria Un modelo comprensivo. Bordón, 71(2), 85-108.

Guitar, M.E. (2011). Aplicaciones contemporáneas de la teoría vigotskiana en educación. Revista de Educación y Desarrollo Social 5(1), 95-113.

Habermas, J. (1989). Teoría de la acción comunicativa: Complementos y estudios previos. Madrid: Cátedra. . (2004). Tiempo de transiciones. Madrid: Trotta 
Helgevold, N. (2016). Teaching as creating space for participation - establishing a learning community in diverse classrooms. Teachers and Teaching, 22(3), 315-328. DOI: $10.1080 / 13540602.2015 .1058590$

Hennissen, P. Beckers, H. y Moerkerke, G. (2017). Linking practice to theory in teacher education: A growth in cognitive structures. Teaching and Teacher Education, 63, 314-325.

Huber, G. L. y Gürtler, L. (2012). AQUAD Six. Manual del programa para analizar datos cualitativos. Tübingen: Ingeborg Huber Verlag). Tübingen

Hughes, J. N. (2012). Teacher-student relationships and school adjustment: progress and remaining challenges. Attachment y Human Development, 14(3), 319-327. DOI: $10.1080 / 14616734.2012 .672288$

Illouz, E. (2010). La salvación del alma moderna. Terapia, emociones y la cultura de la autoayuda. Madrid: Katz

Korthagen, F., Attema, S. y Zwart, R. (2014). Teacher-student contact: Exploring a basic but complicated concept. Teaching and Teacher Education, 40, 22-32. https://doi.org/10.1016/j. tate.2014.01.006

Korthagen, F. A. J. (2004). In search of the essence of a good teacher: towards a more holistic approach in teacher education. Teaching and Teacher Education, 20, 77-97. Recuperado de https://bit.ly/2K1yQYc

. (2010). La práctica, la teoría y la persona en la formación del profesorado. Revista Interuniversitaria de Formación del Profesorado, 68(24), 83-101.

. (2016). Pedagogy of teacher. In J. Loughran y M.L. Lynn. Interbational Handbook of teacher education. Singapore: Springer, 311-347.

Korthagen, F., Kim, Y. y Greene, W. (2013) Teaching and Learning from within: A core Reflection Approach to Quality and Inspiration in Education. New York/London: Routledge

Kvale, S. (2011). Las entrevistas en investigación cualitativa. Madrid: Morat

Lave, J. y Wenger, E. (1991). Situated Learning: Legitimate Peripheral Participation. Cambridge: Cambridge University Press.

Lawrence, J. A. y Dodds, A. E. (2007). MySelf, the Project: Sociocultural interpretations of Young adulthood. En A. J. Valsiner y A. Rosa. The Cambridge Handbook of Sociocultural Psychology. New York: Cambridge University Press.

Lechuga, M. y Ramírez, F. (2015). El proceso de evaluación en la terapia psicomotriz desde el contexto de políticas públicas en chile. Revista iberoamericana de psicomotricidad y técnicas corporales, (40), 128-137.

Leijen, Ä., Allas, R., Pedaste, M. y Knezic, D. (2015). How to Support the Development Of Teachers' Practical Knowledge: Comparing Different Conditions. Procedia Social and Behavioral Sciences, 191, $1205-1212$.

León, J. (2019). Hermenéutica dialógica: claves para pensar la escucha en la educación. Folios, 49, 71-81. DOI: $10.17227 /$ folios.49-9392

Leontiev, A., Luria, A. R y Vigotsky, L. S. (2004). Psicología y pedagogía. Akal: Madrid.

Lipari, L. (2009). Listening otherwise: the voice of ethics. International Journal of listening 23(1). DOI: http://doi.org/10.1080/10904010802591888.

Lozano, J., Ballesta, F. J., Castillo, I. S. y Cerezo, M.C. (2018). El vínculo de la escuela con el territorio: Una experiencia de inclusión educativa. Profesorado Revista de currículum y formación del profesorado, 22(2), 207-226.

Martin, A. Tarnanen, M. y Tynjälä, P. (2018). Exploring teachers' stories of writing: a narrative perspective. Teachers and Teaching theory and practice, 24(6), 690-705. https://doi.org/10.10 80/13540602.2018.1462790.

Matusov, E. (2015). Chronotopes in education: conventional and dialogic. Dialogic Pedagogy: An International Online Journal, Vol. 3, doi: 10.5195/dpj.2015.107. Recuperado de http://dpj.pitt. edu 
Estudios Pedagógicos XLVII N 1: 323-337, 2021

PERCEPCIÓN DOCENTE DE LA NATURALEZA DE LA ESCUCHA PEDAGÓGICA E IMPLEMENTACIÓN DE LAS RELACIONES COMUNICATIVAS EN LA EDUCACIÓN SECUNDARIA

Menéndez Álvarez Hevia, D. (2018). Aproximación crítica a la Inteligencia Emocional como discurso dominante en el ámbito educativo. Revista Española de Pedagogía, 76(269), 7 23. doi: https:// doi.org/10.22550/ REP76 1201801

Monarca, H., Rappoport, S. y Fernández, A. (2012). Factores condicionantes de las trayectorias escolares en la transición entre Enseñanza Primaria y Secundaria. Revista Española de Orientación y Psicopedagogía 23(3), 49-62.

Morin, E. (2009). Introducción al pensamiento Complejo. Madrid: Gedisa.

Neira, M. R., Sierra, B. y Pérez, M. (2018). La competencia comunicativa en el Grado de Maestro/a en Educación Infantil y Primaria. Una propuesta de criterios de desempeño como referenciamarco para su análisis y evaluación. Revista complutense de Educación, 29(3), 881-898.

Nuñez del Río, M. C. y Fontana, M. (2009). Competencias socioemocionales en el aula: características del profesor que favorece la motivación por el aprendizaje en alumnos de enseñanza secundaria obligatoria. Reop, 20(3), 257-269.

Offir, N. (2012). La pedagogía por proyectos en la escuela. Una revisión de sus fundamentos filosóficos y psicológicos. Magis, Revista Internacional de Investigación en Educación, 4(9), 685-707.

Oliveira, A. V., Santos Mafra De Benedictis, N. M., Amorim de Oliveira, L. y Sandes, V. (2018). A contribuição das narrativas autobiográficas para as discussões sobre formação docente. Revista Tempos e Espaços em Educação, São Cristóvão, 11(25) 241-250.

Ortega, P. (2017). La Educación es un encuentro con el otro. Revista Virtual Redipe, 6(8), 25-36.

Ortega, P. y Gárate, A. (2017). Una escuela con rostro humano. Mexicali: Cetys.

Otzen, T. y Manterola, O. (2017). Técnicas de Muestreo sobre una Población a Estudio. Int. J. Morphol, 35(1), 227-232.

Pagès, A. (2016). Actualidad de la hermenéutica como filosofía de la Educación. Revista Española de Pedagogía, 264, 265-281.

Penman, R. y Turnbull, S. (2012). From Listening...to the dialogic realities of participatori democracy. Journal of Media y Cultural Studies, 26(1), 61-72. DOI: http//doi.org/10.1080/10 304312.2012.630145.

Perrenoud, P. (2012). Cuando la escuela pretende preparar la vida. ¿Desarrollar competencias o enseñar otros saberes? Barcelona: Graó.

Phillips, L. y Napan, K. (2016). What's in the 'co'? Tending the tensions in co-creative inquiry in social work education. International Journal of Qualitative Studies in Education, 29(6), 827844. DOI: 10.1080/09518398.2016.1162869

Pizzinato, A. (2009). Psicología cultural. Contribuciones teóricas y fundamentos epistemológicos de las aportaciones de Vigotsky hacia la discusión lingüística de Bakhtín. Universitas Psichologica, 9(1). 255-261

Pons R. M. y Serrano J. M. (2011). La adquisición del conocimiento: una perspectiva cognitiva en el dominio de las matemáticas. Educatio Siglo XXI, 29(2) 117-138.

Pozo, J. I. (2017). Learning beyond the body: from embodied representations to explicitation mediated by external representations. Infancia y Aprendizaje, 40(2), 219-276. DOI: 10.1080/02103702.2017.1306942.

. (2011). La construcción de la identidad docente en psicología cognitiva:del aprendizaje situado a la integración jerárquica. En A.C. Monereo y J. I. Pozo (Eds.). La identidad en psicología de la educación: necesidad y límites. Madrid: Narcea.

Prados, M. M, Gómez del castillo, M. T. y Aguilera Jiménez, A. (2016). Comunidades de aprendizaje: ¿Qué aportan a los voluntarios y voluntarias universitarios? Revista Complutense de Educación, 27(2), 551-570.

Recalcati, M. (2016). La hora de clase. Anagrama: Barcelona

Rivas-Flores, J. I, Cortés, P. y Leite, A. E. (2014). Un enfoque narrativo en la identidad profesional en el profesorado Novel. Tendencias pedagógicas, 24,199-214. 
Rodriguez-Hoyos, C., Calvo-Salvador, A. y Haya-Simón, I. (2014). La tutoría académica en la educación superior. Una investigación a partir de entrevistas y grupos de discusión en la Universidad de Cantabria (España). Revista Complutense de Educación, 26(2), 467-481

Romero, S., Figuera, P., Freixa, M. y Llanes, J. (2019). Adaptabilidad de la carrera en estudiantes universitarios: Un estudio a través de entrevistas autobiográficas. Revista de Investigación Educativa, 37(2), 379-394. DOI: http://dx.do.org/10.6018/rie.37.2.322441

Ruhalahti, S. y Aarnio, H. (2018). Self-paced and dialogical knowledge creation for promoting deep learning: the pilot case in teacher education. Ibero-Americana de Estudos em Educação, 13(1), 291-303.

Sánchez, I. (2018). Aproximación etnográfica a la construcción de identidades femeninas y masculinas en Educación Primaria. Revista Complutense de educación, 29(4), 1025-1039.

Sarceda, M. C. y Rodicio García, M. L. (2018). Escenarios formativos y competencias profesionales en la formación inicial del profesorado. Revista Complutense de Educación, 29(1), 147-164.

Segovia, J. D., Gallego, J. L. y Rodríguez, A. (2013). Percepción del profesorado sobre la competencia comunicativa en estudiantes de Magisterio. Perfiles educativos, 35(142), 54-74.

Sousa, C. (2017). Produção textual e autoria em ambiente virtual de aprendizagem: uma escrita dialógica. Belo Horizonte, 10(2), 308-323.

Tejedor, F. J. (2018). Investigación educativa: la utilidad como criterio social de calidad. Revista de Investigación Educativa, 36(2), 315-330. DOI: http//dx.doi.org/10.6018/rie.36.2.326311

Tenti Fanfani, E. (2010). Los que ponen el cuerpo. El profesor de secundaria en la Argentina actual. Educar em Revista, (1), 37-76. Recuperado el 5 de julio de 2018 desde https://bit.ly/2Mc3GjM

Vigotsky, L. S. A. (1978). Pensamiento y lenguaje, Madrid: Paidós.

Villalta, M. A. (2009). Análisis de la conversación. Una propuesta para el estudio de la interacción didáctica en sala de clase. Estudios Pedagógicos 35(1), 221-238. DOI: 10.4067/S071807052009000100013

Villalta, M. A., Martinic, S., Assael, C. y Aldunate, N. (2018). Presentación de un modelo de análisis de la conversación y experiencias de aprendizaje mediado en la interacción de sala de clase. Revista Educación, 42(1), 1-18.

Wertsch, J. V. (2005). Voices of collective remembering. N.York: Cambridge University Press.

Zhao, K. y Biesta, G. (2012). The moral dimension of lifelong learning: Giddens, Taylor, and the "Reflexive project of the self". Adult Education Quarterly, 62(4), 332-350. 
